PROCEEDINGS OF THE

AMERICAN MATHEMATICAL SOCIETY

Volume 126, Number 6, June 1998, Pages 1845-1850

S 0002-9939(98)04733-9

\title{
EIGENVALUES OF THE FORM VALUED LAPLACIAN FOR RIEMANNIAN SUBMERSIONS
}

\author{
PETER B. GILKEY, JOHN V. LEAHY, AND JEONG HYEONG PARK
}

(Communicated by Christopher Croke)

\begin{abstract}
Let $\pi: Z \rightarrow Y$ be a Riemannian submersion of closed manifolds. Let $\Phi_{p}$ be an eigen $p$-form of the Laplacian on $Y$ with eigenvalue $\lambda$ which pulls back to an eigen $p$-form of the Laplacian on $Z$ with eigenvalue $\mu$. We are interested in when the eigenvalue can change. We show that $\lambda \leq \mu$, so the eigenvalue can only increase; and we give some examples where $\lambda<\mu$, so the eigenvalue changes. If the horizontal distribution is integrable and if $Y$ is simply connected, then $\lambda=\mu$, so the eigenvalue does not change.
\end{abstract}

If $M$ is a closed Riemannian manifold, let $E\left(\lambda, \Delta_{M}^{p}\right)$ be the eigenspace of the Laplacian $\Delta_{M}^{p}:=d \delta+\delta d$ for the eigenvalue $\lambda$ on the space of smooth $p$-forms $C^{\infty}\left(\Lambda^{p} M\right)$. Let $\pi: Z \rightarrow Y$ be a Riemannian submersion of closed manifolds. Pullback defines a natural map $\pi^{*}$ from $C^{\infty}\left(\Lambda^{p} Y\right)$ to $C^{\infty}\left(\Lambda^{p} Z\right)$. We are interested in examples where an eigenform on $Y$ pulls back to an eigenform on $Z$ with a different eigenvalue. Let $\mathcal{V}$ and $\mathcal{H}$ be the vertical and horizontal distributions of $\pi$. We say that $\mathcal{H}$ is an integrable $S L$ (Special Linear) distribution if $\mathcal{H}$ is integrable and if there exists a measure $\nu$ on the fibers of $\pi$ so that the Lie derivative $\mathcal{L}_{H} \nu$ vanishes for all horizontal lifts $H$; this means that the transition functions of the fibration can be chosen to have Jacobian determinant 1.

Theorem 1. Let $\pi: Z \rightarrow Y$ be a Riemannian submersion of closed manifolds. Let $\Phi_{p} \in E\left(\lambda, \Delta_{Y}^{p}\right)$ and $\pi^{*} \Phi_{p} \in E\left(\mu, \Delta_{Z}^{p}\right)$. If $\mathcal{H}$ is an integrable $S L$ distribution, $\lambda=\mu$.

We will show in Lemma 6 that if $Y$ is simply connected and if $\mathcal{H}$ is integrable, then $\mathcal{H}$ is an integrable $S L$ distribution and therefore eigenvalues do not change. This shows the fundamental role that the curvature tensor plays in this subject. We note that the Godbillon-Vey class of the foliation $\mathcal{H}$ vanishes if $\mathcal{H}$ is an integrable $S L$ distribution.

In the general setting, we show that if eigenvalues change, they can only increase.

Theorem 2. Let $\pi: Z \rightarrow Y$ be a Riemannian submersion of closed manifolds. Let $\Phi_{p} \in E\left(\lambda, \Delta_{Y}^{p}\right)$ and $\pi^{*} \Phi_{p} \in E\left(\mu, \Delta_{Z}^{p}\right)$. Then $\lambda \leq \mu$.

It is not difficult to use the maximum principle to show that eigenvalues cannot change if $p=0$. It is not known if eigenvalues can change if $p=1$. Eigenvalues can change if $p \geq 2$.

Received by the editors May 20, 1996 .

1991 Mathematics Subject Classification. Primary 58G25.

Key words and phrases. Riemannian submersion, eigenvalues, Laplacian.

The first author's research was partially supported by the NSF (USA); the third author's, by BSRI-96-1425, the Korean Ministry of Education. 
Theorem 3. Let $0 \leq \lambda<\mu$. If $p \geq 2$, there exists a Riemannian submersion $\pi: Z \rightarrow Y$ of closed manifolds so that $\pi$ has totally geodesic fibers and so that there exists $\Phi_{p} \in E\left(\lambda, \Delta_{Y}^{p}\right)$ with $\pi^{*} \Phi_{p} \in E\left(\mu, \Delta_{Z}^{p}\right)$.

Here is a brief guide to the paper. We first review the spectral geometry of Riemannian submersions. Next we study the geometry of a Riemannian submersion with integrable horizontal distribution and prove Theorem 1 . Then we discuss fiber products and give the proof of Theorem 2. The proofs of these results are similar in nature and use the observation that the Laplacian is a non-negative operator. We conclude the paper by using the Hopf fibration to prove Theorem 3; other examples where eigenvalues change may be found in [GLP], [GLPa] and in Muto [Mu], [Mua]. It is a pleasant task to acknowledge helpful conversations with Juha Pohjanpelto.

We adopt the following notational conventions. Let $\pi: Z \rightarrow Y$ be a Riemannian submersion of closed manifolds. We shall use capital letters for tensors on $Y$ and lower case letters for tensors on $Z$. Let $\left\{e_{i}\right\},\left\{e^{i}\right\},\left\{f_{a}\right\}$ and $\left\{f^{a}\right\}$ be local orthonormal frames for $\mathcal{V}, \mathcal{V}^{*}, \mathcal{H}$, and $\mathcal{H}^{*}$. Let $\operatorname{ext}(\xi)$ and $\int(\xi)$ denote exterior multiplication and the dual interior multiplication by the covector $\xi$. We adopt the Einstein convention and sum over repeated indices. Let

$$
\begin{aligned}
& \theta:=-g_{Z}\left(e_{i},\left[e_{i}, f_{a}\right]\right) f^{a}, \omega_{a b i}:=\frac{1}{2} g_{Z}\left(e_{i},\left[f_{a}, f_{b}\right]\right), \\
& \mathcal{E}:=\omega_{a b i} \operatorname{ext}\left(e^{i}\right) \operatorname{int}\left(f^{a}\right) \operatorname{int}\left(f^{b}\right) .
\end{aligned}
$$

The fibers of $\pi$ are minimal if and only if the unnormalized mean covector $\theta$ is zero. The horizontal distribution $\mathcal{H}$ is integrable if and only if the curvature $\omega$ is zero.

Theorem 4. Let $\pi: Z \rightarrow Y$ be a Riemannian submersion of closed manifolds.

(a) We have $\Delta_{Z}^{p} \pi^{*}-\pi^{*} \Delta_{Y}^{p}=\left\{d\left(\int(\theta)+\mathcal{E}\right)+\left(\int(\theta)+\mathcal{E}\right) d\right\} \pi^{*}$.

(b) If $p=0$, then the following conditions are equivalent:

i) We have $\Delta_{Z}^{0} \pi^{*}=\pi^{*} \Delta_{Y}^{0}$.

ii) For all $\lambda \in \mathbb{R}$, there exists $\mu(\lambda) \in \mathbb{R}$ so $\pi^{*} E\left(\lambda, \Delta_{Y}^{0}\right) \subseteq E\left(\mu(\lambda), \Delta_{Z}^{0}\right)$.

iii) The fibers of $\pi$ are minimal.

(c) If $1 \leq p \leq \operatorname{dim}(Y)$, then the following conditions are equivalent:

i) We have $\Delta_{Z}^{p} \pi^{*}=\pi^{*} \Delta_{Y}^{p}$.

ii) For all $\lambda \in \mathbb{R}$, there exists $\mu(\lambda) \in \mathbb{R}$ so $\pi^{*} E\left(\lambda, \Delta_{Y}^{p}\right) \subseteq E\left(\mu(\lambda), \Delta_{Z}^{p}\right)$.

iii) The fibers of $\pi$ are minimal and $\mathcal{H}$ is integrable.

Remark 5. Assertion (a) of Theorem 4 is the fundamental formula in this subject. If $p=0$, it follows from the work of Watson [Wa] and if $p>0$, it follows from the work of Goldberg and Ishihara [GoIs]; these authors used it to prove the equivalence of i) and iii) in assertions (b) and (c). We also refer to [GP] for a slightly different proof of assertion (a) as well as a proof of the equivalence of i) and ii) in assertions (b) and (c). Suppose the pull-back of every eigen $p$-form on the base is an eigen $p$ form on the total space. We then have $\pi^{*} E\left(\lambda, \Delta_{Y}^{p}\right) \subset E\left(\mu\left(\lambda, \Delta_{Z}^{p}\right)\right)$ as the eigenvalue in question must be constant on any given eigenspace. Assertions (b) and (c) then show $\mu(\lambda)=\lambda$; the eigenvalue cannot change.

We begin the proof of Theorem 1 by showing:

Lemma 6. Let $\pi: Z \rightarrow Y$ be a Riemannian submersion of closed manifolds. Assume that $\mathcal{H}$ is integrable.

(a) We can find local coordinates $z=(x, y)$ on $Z$ so that $\pi(x, y)=y$ and so that $d s_{Z}^{2}=g_{i j}(x, y) d x^{i} \circ d x^{j}+h_{a b}(y) d y^{a} \circ d y^{b}$. 
(b) Let $g_{X}=\operatorname{det}\left(g_{i j}\right)^{1 / 2}$. Then $\theta=-d_{Y} \ln \left(g_{X}\right)$.

(c) If $\pi_{1}(Y)=0$, then we may decompose $Z=X \times Y$ such that $\pi(x, y)=y$. Furthermore, $\mathcal{H}$ is an integrable $S L$ distribution.

(d) If we define a new metric on $Z$ by replacing the vertical metric $d s_{\mathcal{V}}^{2}$ on the vertical distribution by the rescaled metric $e^{-2 \alpha / \operatorname{dim}(X)} d s_{\mathcal{V}}^{2}$ for $\alpha \in C^{\infty}(Z)$, then we have $\theta_{\alpha}=\theta+\partial_{Y} \alpha$.

(e) If $\mathcal{H}$ an integrable $S L$ distribution, then there exists $\alpha \in C^{\infty}(Z)$ so that $\theta=-d_{Y} \alpha$.

Proof. We have that $Z$ is a twisted product over $Y$ with fiber $X$. Choose local coordinates $y=\left(y^{a}\right)$ defined on a neighborhood $\mathcal{O}$ of any point $y_{0}$ in $Y$. Let $\tilde{f}_{a}$ be the horizontal lift of the coordinate vector fields $\partial / \partial y_{a}$ from $Y$ to $Z$ over $\pi^{-1}(\mathcal{O})$; this is not an orthonormal frame. Since $\mathcal{H}$ is integrable, we have $\left[\tilde{f}_{a}, \tilde{f}_{b}\right] \in \mathcal{H}$. We use the equation $\pi_{*}\left(\left[\tilde{f}_{a}, \tilde{f}_{b}\right]\right)=\left[\partial / \partial y_{a}, \partial / \partial y_{b}\right]=0$ to see that $\left[\tilde{f}_{a}, \tilde{f}_{b}\right]=0$. Choose local coordinates $x=\left(x^{i}\right)$ for the fiber over $y_{0}$ near a point $z_{0} \in \pi^{-1}\left(y_{0}\right)$. We use the Frobenius theorem to extend $x$ to a system of coordinates $z=(x, w)$ on a neighborhood of $z_{0}$ so that $\tilde{f}_{a}=\partial / \partial w_{a}$. The projections of the integral curves of the vector fields $\partial / \partial w_{a}$ are the integral curves of the vector fields $\partial / \partial y_{a}$. Therefore $y=\pi(x, w)=w$ and $\pi$ is projection on the second factor. Since $\mathcal{V}=T X=$ $\operatorname{span}\left\{\partial / \partial x^{i}\right\}$ is perpendicular to $\mathcal{H}=T Y=\operatorname{span}\left\{\partial / \partial w^{a}\right\}$ and since $\pi_{*}$ is an isometry from $\mathcal{H}$ to $T Y$, the metric locally has the form given in assertion (a). If $d s_{M}^{2}=g_{r s} d u^{r} \circ d u^{s}$, let $g_{M}=\operatorname{det}\left(g_{r s}\right)^{1 / 2}$. Then $\Delta_{M}^{0}=-g_{M}^{-1} \partial_{r} g_{M} g^{r s} \partial_{s}$. Express the metric on $Z$ locally as in assertion (a). Then

$$
\operatorname{int}(\theta) d \pi^{*} \Phi_{0}=\left(\Delta_{Z}^{0} \pi^{*}-\pi^{*} \Delta_{Y}^{0}\right) \Phi_{0}=-\int\left(g_{X}^{-1} d_{Y} g_{X}\right) d \pi^{*} \Phi_{0}
$$

for any $\Phi_{0} \in C^{\infty}(Y)$, where $g_{X}=\operatorname{det}\left(g_{i j}(x, y)\right)^{1 / 2}$. This shows $\theta=-d_{Y} \ln \left(g_{X}\right)$. Assertion (b) now follows.

Assume the base $Y$ is simply connected. Parallel translation along a curve defines a diffeomorphism from the fiber of $\pi$ at the initial point to the fiber of $\pi$ at the terminal point of the curve. We have that this diffeomorphism is independent of the curve and gives a global splitting $Z=X \times Y$ so that $\pi(x, y)=y$ and $\mathcal{H}=\operatorname{span}\left\{\partial / \partial y^{a}\right\}$, where $X$ is the fiber over the basepoint. If $\nu$ is any smooth measure on $X$, then $\mathcal{L}_{H} \nu=0$ for the horizontal lift of any vector field on the base $Y$, so $\mathcal{H}$ is an integrable $S L$ distribution. Assertions (c) and (d) now follow.

Suppose that $\mathcal{H}$ is an integrable $S L$ distribution. Let $\nu_{X}$ be a measure on the fibers so $\mathcal{L}_{H} \nu_{X}=0$ for all the horizontal lifts $H$. Let

$$
\mathrm{dvol}_{Z}=e^{\alpha} \nu_{X} \mathrm{dvol}_{Y} \text { and } \nu_{X}=e^{\beta} d x^{1} \ldots d x^{\operatorname{dim}(X)} .
$$

Then $\alpha$ is globally defined, $\beta$ is locally defined, and $g_{X}=e^{\alpha+\beta}$. Since $\mathcal{L}_{\partial / \partial y} \nu_{X}=0$, $\beta$ is independent of the base. Therefore $\theta=-d_{Y} \ln \left(g_{X}\right)=-d_{Y} \alpha$.

Proof of Theorem 1. Let $\mathcal{H}$ be an integrable $S L$ foliation. Let $\Phi_{p} \in E\left(\lambda, \Delta_{Y}^{p}\right)$ with $\pi^{*} \Phi_{p} \in E\left(\mu, \Delta_{Z}^{p}\right)$. Since the curvature vanishes, we may apply Theorem 4 to get

$$
\begin{gathered}
(\mu-\lambda) \pi^{*} \Phi_{p}(x, y)=\left(\Delta_{Z}^{p} \pi^{*} \Phi_{p}-\pi^{*} \Delta_{Y}^{p} \Phi_{p}\right)(x, y) \\
=\left(d_{Z} \int(\theta) \pi^{*} \Phi_{p}+\int(\theta) \pi^{*} d_{Y} \Phi_{p}\right)(x, y) .
\end{gathered}
$$

Choose $\alpha \in C^{\infty}(Z)$ so that $\theta=-d_{Y} \alpha$. We define a new metric $d s_{Z}^{2}(\epsilon)$ on $Z$ by replacing the metric $d s_{\mathcal{V}}^{2}$ on the vertical distribution by $e^{2 \epsilon \alpha / \operatorname{dim}(X)} d s_{\mathcal{V}}^{2}$. We apply 
Lemma 6 to obtain $\theta(\epsilon)=(1+\epsilon) \theta$. Thus $\pi^{*} \Phi_{p} \in E\left(\mu(\epsilon), \Delta_{Z(\epsilon)}^{p}\right)$, where we set $\mu(\epsilon)-\lambda=(1+\epsilon)(\mu-\lambda)$. Since the Laplacian is a non-negative operator, for any $\epsilon \in \mathbb{R}$ we have $\mu(\epsilon)=\lambda+(1+\epsilon)(\mu-\lambda) \geq 0$. This implies $\mu=\lambda$.

Definition 7 (Fiber products). Let $\pi_{U}: U \rightarrow Y$ and $\pi_{V}: V \rightarrow Y$ be Riemannian submersions of closed manifolds. Denote the horizontal and vertical distributions by $\mathcal{H}_{U}, \mathcal{H}_{V}, \mathcal{V}_{U}$, and $\mathcal{V}_{V}$. Let

$$
W:=\left\{w=(u, v) \in U \times V: \pi_{U}(u)=\pi_{V}(v)\right\} .
$$

We identify $T(U \times V)=T(U) \oplus T(V)$ and embed $T(W)$ in $T(U \times V)$. Define $\pi_{W}: W \rightarrow Y$ by $\pi_{W}(w):=\pi_{U}(u)=\pi_{V}(v)$. Let $\mathcal{V}_{W}(w):=\mathcal{V}_{U}(u) \oplus \mathcal{V}_{V}(v)$ and

$$
\mathcal{H}_{W}(w):=\left\{(\xi, \eta) \in \mathcal{H}_{U}(u) \oplus \mathcal{H}_{V}(v):\left(\pi_{U}\right)_{*} \xi=\left(\pi_{V}\right)_{*} \eta\right\} .
$$

We define a new metric on $W$ by requiring that $\mathcal{H}_{W}, \mathcal{V}_{U}$, and $\mathcal{V}_{V}$ are orthogonal, that the metrics on $\mathcal{V}_{U}$ and $\mathcal{V}_{V}$ are induced from the metrics on $U$ and on $V$, and that $\left(\pi_{W}\right)_{*}: \mathcal{H}_{W} \rightarrow T Y$ is an isometry. The metric on $\mathcal{H}_{W}$ differs from the induced metric by a factor of $2^{-1 / 2}$. Let $\pi_{1}(u, v)=u$ and $\pi_{2}(u, v)=v$. Then $\pi_{1}: W \rightarrow U$, $\pi_{2}: W \rightarrow V$, and $\pi_{W}: W \rightarrow Y$ are Riemannian submersions. Let $f_{a, U}$ and $f_{a, V}$ be the horizontal lifts of $F_{a}$ with respect to the submersions $\pi_{U}$ and $\pi_{V}$. Then $f_{a, W}:=f_{a, U}+f_{a, V}$ is the horizontal lift of $F_{a}$ with respect to the submersion $\pi_{W} ; f_{a, W}$ is also the horizontal lift of $f_{a, U}$ and $f_{a, V}$ to $W$ with respect to the submersions $\pi_{1}$ and $\pi_{2}$. Let $\left\{e_{i, U}\right\}$ and $\left\{e_{\alpha, V}\right\}$ be local orthonormal frames for the vertical distributions $\mathcal{V}_{U}$ and $\mathcal{V}_{V}$ of the submersions $\pi_{U}$ and $\pi_{V}$. Then $\left\{e_{i, U}, e_{\alpha, V}\right\}$ is a local orthonormal frame for the vertical distribution $\mathcal{V}_{W}$ of the submersion $\pi_{W}$. Furthermore $\left\{e_{i, U}\right\}$ and $\left\{e_{\alpha, V}\right\}$ are local orthonormal frames for the vertical distributions $\mathcal{V}_{U}$ and $\mathcal{V}_{V}$ of the submersions $\pi_{2}$ and $\pi_{1}$.

Example 8. Suppose that $U$ and $V$ are vector bundles over $Y$ with given fiber metrics and Riemannian connections. This defines Riemannian metrics on $U$ and $V$ so that the projections $\pi_{U}$ and $\pi_{V}$ are Riemannian submersions; if $\gamma$ is a curve in $Y$, then $s_{\gamma}$ is the horizontal lift of $\gamma$ if and only if $s_{\gamma}$ is parallel along $\gamma$. Then $W=U \oplus V$ is the Whitney sum vector bundle, and the metric on $W$ is defined by the Whitney sum connection and the Whitney sum fiber metric.

Lemma 9. Adopt the notation of Definition $\%$

(a) We have $\theta_{W}=\pi_{1}^{*} \theta_{U}+\pi_{2}^{*} \theta_{V}$ and $\mathcal{E}_{W} \pi_{W}^{*}=\pi_{1}^{*} \mathcal{E}_{U} \pi_{U}^{*}+\pi_{2}^{*} \mathcal{E}_{V} \pi_{V}^{*}$.

(b) If $\Phi_{p} \in E\left(\lambda, \Delta_{Y}^{p}\right)$, if $\pi_{U}^{*} \Phi_{p} \in E\left(\lambda+\epsilon_{U}, \Delta_{U}^{p}\right)$, and if $\pi_{V}^{*} \Phi_{p} \in E\left(\lambda+\epsilon_{V}, \Delta_{V}^{p}\right)$, then we have $\pi_{W}^{*} \Phi_{p} \in E\left(\lambda+\epsilon_{U}+\epsilon_{V}, \Delta_{W}^{p}\right)$.

Proof. We note that pull-back commutes with exterior and interior multiplication. We prove assertion (a) by computing:

$$
\begin{aligned}
& \theta_{W}=-\left\{g_{W}\left(e_{i, U},\left[e_{i, U}, f_{a, W}\right]\right)+g_{W}\left(e_{\alpha, V},\left[e_{\alpha, V}, f_{a, W}\right]\right)\right\} \pi_{W}^{*}\left(F^{a}\right) \\
& \quad=-\left\{g_{U}\left(e_{i, U},\left[e_{i, U}, f_{a, U}\right]\right)+g_{V}\left(e_{\alpha, V},\left[e_{\alpha, V}, f_{a, V}\right]\right)\right\} \pi_{W}^{*}\left(F^{a}\right) \\
& \quad=\pi_{1}^{*} \theta_{U}+\pi_{2}^{*} \theta_{V}, \\
& {\left[f_{a, W}, f_{b, W}\right]=\left[f_{a, U}, f_{b, U}\right]+\left[f_{a, V}, f_{b, V}\right],} \\
& \omega_{W i a b}=g_{W}\left(e_{i, W},\left[f_{a, W}, f_{b, W}\right]\right)=g_{U}\left(e_{i, U},\left[f_{a, U}, f_{b, U}\right]\right)=\omega_{U i a b}, \\
& \omega_{W \alpha a b}=g_{W}\left(e_{\alpha, W},\left[f_{a, W}, f_{b, W}\right]\right)=g_{V}\left(e_{\alpha, V},\left[f_{a, V}, f_{b, V}\right]\right)=\omega_{V \alpha a b} .
\end{aligned}
$$


We use assertion (a) and Theorem 4 to prove (b) by computing:

$$
\begin{aligned}
\left(\epsilon_{U}+\epsilon_{V}\right) \pi_{W}^{*} \Phi_{p} & =\pi_{1}^{*}\left\{\Delta_{U}^{p} \pi_{U}^{*}-\pi_{U}^{*} \Delta_{Y}^{p}\right\} \Phi_{p}+\pi_{2}^{*}\left\{\Delta_{V}^{p} \pi_{V}^{*}-\pi_{V}^{*} \Delta_{Y}^{p}\right\} \Phi_{p} \\
& =\left(\Delta_{W}^{p} \pi_{W}^{*}-\pi_{W}^{*} \Delta_{Y}^{p}\right) \Phi_{p} . \quad \square
\end{aligned}
$$

Proof of Theorem 2. Suppose that $\pi: Z \rightarrow Y$ is a Riemannian submersion of closed manifolds. Let $0 \neq \Phi_{p} \in E\left(\lambda, \Delta_{Y}^{p}\right)$ and $\pi^{*} \Phi_{p} \in E\left(\lambda+\epsilon, \Delta_{Z}^{p}\right)$. Let $Z_{0}=Z$, and inductively let $Z_{n}=W\left(Z_{n-1}, Z_{n-1}\right)$ be the fiber product of $Z_{n-1}$ with itself and let $\pi_{n}: Z_{n} \rightarrow Y$ be the associated projection. We use Lemma 9 (b) and induction to see

$$
\pi_{n}^{*} \Phi_{p} \in E\left(\lambda+2^{n} \epsilon, \Delta_{Z_{n}}^{p}\right) .
$$

Since the Laplacian on $Z_{n}$ is non-negative, $\lambda+2^{n} \epsilon \geq 0$. This implies $\epsilon \geq 0$.

Proof of Theorem 3. Let $\pi: S^{3} \rightarrow S^{2}$ be the Hopf fibration. Let $\Phi_{2}$ be the volume form on $S^{2}$ with respect to the standard metric $g$. Then $\Phi_{2} \in E\left(0, \Delta_{S^{2}}^{2}\right)$. In [GLP, Theorem 2.3], we constructed a metric $\tilde{g}(\alpha)$ on $S^{3}$ so that $\pi$ is a Riemannian submersion with totally geodesic fibers from $\left(S^{3}, \tilde{g}(\alpha)\right)$ to $\left(S^{2}, g\right)$ and so that $\pi^{*}\left(\Phi_{2}\right) \in E\left(\alpha^{2}, \Delta_{S^{3}, \tilde{g}(\alpha)}^{2}\right)$. This completes the proof if $p=2$ and $\lambda=0$. If $0<\lambda<\mu$, let $\lambda=\alpha^{2}$ and $\mu=\alpha^{2}+\beta^{2}$. Let $W=W(\alpha, \beta)$ be the fiber product of $\left(S^{3}, \tilde{g}(\alpha)\right)$ and $\left(S^{3}, \tilde{g}(\beta)\right)$, where $\lambda=\alpha^{2}$. Let $\pi_{\alpha}: W \rightarrow S^{3}$ and let $\pi_{W}: W \rightarrow S^{2}$. By Lemma 9,

$$
\pi^{*} \Phi_{2} \in E\left(\alpha^{2}, \Delta_{S^{3}, \tilde{g}(\alpha)}^{2}\right)
$$

and

$$
\pi_{\alpha}^{*} \pi^{*} \Phi_{2}=\pi_{W}^{*} \Phi_{2} \in E\left(\alpha^{2}+\beta^{2}, \Delta_{W}^{2}\right) .
$$

This completes the proof if $p=2$; the general case now follows by taking suitable Riemannian products.

This constructs examples where the pull-back of a non-trivial eigenform is an eigenform and where eigenvalues can change. Conversely, in [GLP, Theorem 2.3], we constructed a right invariant metric $\tilde{g}_{3}$ on the Lie group $S^{3}$ so the Hopf map $\pi$ is a Riemannian submersion from $\left(S^{3}, \tilde{g}_{3}\right)$ to $S^{2}$ with the standard metric and so that $\pi^{*} \Phi_{p} \in E\left(\lambda, \Delta_{S^{3}}^{p}\right)$ for $\Phi_{p} \in C^{\infty} \Lambda^{p} S^{2}$ implies $p=0, \Phi_{0}=c$, and $\lambda=0$. This provides an example where only the constant function on $Y$ pulls back to an eigenform on $Z$; this is generically the situation for a Riemannian submersion.

\section{REFERENCES}

[GLP] P. B. Gilkey, J. V. Leahy, and J. H. Park, The spectral geometry of the Hopf fibration, J. Phys. A 29 (1996), 5645-5656. CMP 97:04

[GLPa] , The eigenforms of the complex Laplacian for a Hermitian submersion, preprint.

[GP] P. B. Gilkey and J. H. Park, Riemannian submersions which preserve the eigenforms of the Laplacian, Illinois J. Math. 40 (1996), 194-201. MR 97h:58173

[GoIs] S. I. Goldberg and T. Ishihara, Riemannian submersions commuting with the Laplacian, J. Diff. Geo. 13 (1978), 139-144. MR 80c:53047

$[\mathrm{Mu}]$ Y. Muto, Some eigenforms of the Laplace-Beltrami operators in a Riemannian submersion, J. Korean Math. Soc. 15 (1978), 39-57. MR 81j:58085 
[Mua] L Riemannian submersion and the Laplace-Beltrami operator, Kodai Math. J. 1 (1978), 329-338. MR 80a:53064

[Wa] B. Watson, Manifold maps commuting with the Laplacian, J. Diff. Geo. 8 (1973), 85-94. MR 51:1671

Department of Mathematics, University of Oregon, Eugene, Oregon 97403

E-mail address: gilkey@math.uoregon.edu

E-mail address: leahy@math.uoregon.edu

Department of Mathematics, Honam University, Seobongdong 59, Kwangsanku, Kwanguu, 506-090 South Korea

E-mail address: jhpark@honam.honam.ac.kr 